\title{
Minimal expression of myotonic dystrophy: a clinical and molecular analysis
}

\author{
W Reardon, H G Harley, J D Brook, S A Rundle, S Crow, P S Harper, D J Shaw
}

\begin{abstract}
A clinical and molecular study is reported of 83 patients considered to be minimally affected with myotonic dystrophy (DM). These had been identified in three ways: 60 subjects were identified on clinical grounds and were divided into those with and those without neuromuscular involvement (groups I and II); nine subjects were at high risk of carrying the DM gene but had a normal phenotype (group III); and 14 were parents of definitely affected patients where neither parent showed clinical abnormalities (group IV). PCR analysis of the CTG repeat in the DM gene showed a range of 70 to 230 repeats for the younger at risk patients in group III, while the asymptomatic gene carriers in group IV had 53 to 60 repeats. The sensitivity of diagnosis by EMG was found to be $39 \%$. For ophthalmic signs this was $97 \cdot 5 \%$. This suggests that assignment on the basis of minimal clinical features carries a significant error. Molecular analysis, in conjunction with established clinical investigations, should prove valuable in the identification and exclusion of minimal myotonic dystrophy.
\end{abstract}

(f Med Genet 1992;29:770-3)

The involvement of many different systems in myotonic dystrophy (DM) is reflected by an extraordinary variability in the clinical presentation of the condition from one patient to the next. Although the classical clinical picture is one of myotonia with a variable but characteristic distribution of muscle weakness, clinicians familiar with the condition recognise that clear manifestations of muscle disease are not essential to a positive diagnosis in all cases. The diagnostic value of extramuscular features in identifying gene carriers has been recognised since Fleischer confirmed Curschmann's observation that cataract was associated with myotonic dystrophy. ${ }^{12}$ Moreover, the striking relationship between clinical features and age at onset of disease, with the mildest cases tending to be seen in the oldest generation of a family, has been a constant feature of several studies of the condition. ${ }^{3}$ This phenomenon of 'anticipation', where the top generation of a pedigree is identifiable mainly through ophthalmic manifestations, has allowed the recognition of a group of subjects who have been termed 'minimal' gene carriers. ${ }^{4}$ Such patients show little or no evidence of muscle involvement and are generally unaware of the significance of their own features until the family study which follows diagnosis of the classical or congenital form of myotonic dystrophy in a child or a grandchild. It is rare for these minimally expressing gene carriers to be diagnosed as having myotonic dystrophy in isolation.

Minimal expression of the DM phenotype is most commonly diagnosed through ophthalmic signs, of which a wide variety have been documented. ${ }^{3}$ These range from the classical polychromatic, iridescent subcapsular opacities of the subclinical early cataract to mature cataract, which may have been removed by the time its wider significance is recognised. The lens opacities are best documented by slit lamp examination, but, even in the presence of polychromasia, are not absolutely specific, making the definitive identification of minimal gene carriers not always possible on ophthalmic features only.

Evidence of subclinical muscle expression of myotonic dystrophy may also be demonstrable on EMG examination in the form of increased electrical activity, myotonic runs, and dystrophic changes, but not all minimally expressing gene carriers have positive EMG findings, so that this approach has a small but appreciable false negative rate.

The identification of close linkage between 19q markers and the myotonic dystrophy locus has formed the basis of predictive testing for the condition. Attention to the accurate identification of minimally expressing gene carriers has been an important aspect of the optimal application of these tests. ${ }^{56}$ Results obtained by linkage have been compared with clinical findings in the subjects seeking predictive testing for myotonic dystrophy, suggesting that 8 to $10 \%$ of patients with negative clinical, EMG, and ophthalmic findings have inherited the high risk haplotype ${ }^{67}$; this suggests a sensitivity of approximately $90 \%$ in identifying subclinical gene carriers by clinical and electrophysiological means.

The molecular basis of myotonic dystrophy has recently been elucidated and the disorder traced to an unstable triplet repeat (dCTG) in the $3^{\prime}$ untranslated region of the gene, which has increased in copy number, or 'expanded', in myotonic dystrophy. ${ }^{8-11}$ Preliminary data suggest a relationship between the expansion of the unstable sequence and the degree of clinical manifestation of the disorder, with minimally affected patients having least expansion of DNA. ${ }^{12}$ In order to verify this observation, a large series of minimally expressing gene carriers of myotonic dystrophy needs to be studied. This paper reports clinical and molecular data on such a series of patients and 
examines the relationship between the molecular defect and the clinical findings in patients with minimal expression of the myotonic dystrophy gene.

\section{Materials and methods}

CLINICAL ASPECTS

From the records of the 400 myotonic dystrophy pedigrees known to this department, 83 patients were identified as being likely to have minimal features of myotonic dystrophy. These were divided into four groups as follows.

Patients with minimal feature of myotonic dystrophy, considered definitely to be affected on clinical grounds (groups $I$ and II)

Sixty such patients ( 39 males and 21 females) were identified. All had a first degree relative definitely affected with myotonic dystrophy. Clinical data as well as EMG and slit lamp examination results were obtained by personal examination in the case of patients seen in this department or by the investigations of the clinician responsible for cases from other regions.

These 60 patients were separated into two groups on the basis of absence (group I, $n=42$, $28 \mathrm{M}+14 \mathrm{~F}$ ) or presence (group II, $\mathrm{n}=18$, $11 \mathrm{M}+7 \mathrm{~F}$ ) of signs of muscle disease. Patients reporting muscle symptoms before the age of 40 years were excluded from the study.

Patients predicted to be at high risk on linkage but at low risk clinically (group III)

In contrast to other groups, these subjects were mostly young, having been seen principally for genetic counselling and assessment of their genetic status. Nine patients (five males and four females) were identified in whom there was a discrepancy between status as predicted by linked markers and clinical investigation. All were at $50 \%$ prior risk of carrying the gene and had had a normal clinical examination with supplementary investigations being performed in most cases.

Parents of myotonic dystrophy patients showing no detectable abnormality (group IV)

Eight families were identified where the gene carrier in the parental or grandparental generation of a case of known classical or congenital myotonic dystrophy could not be identified. In all cases this gave rise to difficulty in processing predictive tests for other family members. Clinical examination was reported to be normal in all cases. Although eight families were included, data are available on only 14 subjects owing to one partner being dead in two instances.

PCR ANALYSIS

DNA was isolated from whole blood by standard procedure ${ }^{13}$ and stored in $1 \times$ TE buffer; $1 \mu \mathrm{l}$ of this was then used for PCR analysis, corresponding to approximately $100 \mathrm{ng}$ of DNA. The PCR analysis was performed as follows: $25 \mu \mathrm{l}$ reactions were set up using standard PCR conditions $(50 \mathrm{mmol} / 1 \mathrm{KCl}$, $1.5 \mathrm{mmol} / 1 \mathrm{MgCl}_{2}, 10 \mathrm{mmol} / 1$ Tris (pH 8.3), $200 \mu \mathrm{mol} / 1 \mathrm{dNTPs}, 1 \mu \mathrm{mol} / 1$ of each primer, and $100 \mathrm{ng}$ template DNA). PCR reactions were carried out in a Hybaid thermal cycler. Cycling conditions were as follows: $1 \times$ three minutes at $94^{\circ} \mathrm{C}, 30 \times$ one minute 30 seconds at $94^{\circ} \mathrm{C}$, one minute at $62^{\circ} \mathrm{C}$, two minutes at $72^{\circ} \mathrm{C}, 1 \times 10$ minutes at $72^{\circ} \mathrm{C}$. Samples were run out on $3 \%$ agarose gels to estimate the number of CTG repeats, with appropriate markers for sizing the amplified product, and classified as normal (five to 30 repeats) or DM specific (50 or more repeats). The primers for the reaction were oligonucleotides 101 and $102^{11}$ which have the following DNA sequence: 101, CTT CCC AGG CCT GCA GTT TGC CCA TC: 102, GAA CGG GGC TCG AAG GGT CCT TGT AGC.

Any sample having only one band by PCR analysis was analysed by Southern blotting to determine whether it had an expanded DNA region $^{8}$ too large to be detectable under the PCR conditions described.

\section{Results}

The results of the PCR analysis of the myotonic dystrophy gene in the various groups of patients are presented in table 1 . The series comprised 83 patients, all of whom had at least one first degree relative with myotonic dystrophy. A result was obtained for 77 ; the remaining six are listed as results outstanding, owing to unavailability of DNA. Molecular analysis of the 77 subjects was done primarily by PCR to estimate the number of copies of the CTG repeat, and by Southern blot analysis to clarify ambiguous results. Three categories of result were possible from the PCR analysis: (1) heterozygotes with a normal band and an abnormally expanded band, (2) subjects with a single normal band, and (3) heterozygotes with two normal bands. Within each of the patient groups, the results are listed according to these

Table 1 Results of PCR analysis.

\begin{tabular}{lccccc}
\hline & Group I & Group II & Group III & Group IV & Total \\
\hline Total no of patients & 42 & 18 & 9 & 14 & 83 \\
Results outstanding & 4 & 1 & 1 & 0 & 6 \\
Subjects with one normal and one abnormal band & 32 & 12 & 5 & 7 & 56 \\
Subjects with one normal band only on PCR & $4(1)^{*}$ & $3(1)^{*}$ & 1 & 2 & 10 \\
Subjects heterozygous for normal alleles & 2 & 2 & 2 & 5 & 11 \\
Size range of DM alleles (CTG) & $53-160$ & $53-160$ & $70-230$ & $53-60$ & \\
\hline
\end{tabular}

* No of patients with a DM specific band identifiable on Southern blot. 
three categories. The correlations of these results with clinical parameters are presented in tables 2 and 3.

In addition to a normal allele, 56 patients $(71 \%)$ showed an expanded allele in the disease range at the myotonic dystrophy locus. Ten $(13 \%)$ patients had only one allele identifiable by PCR and this allele was within the normal size range. Southern blot analysis of these 10 subjects showed the following. For the four patients from group I, three subjects (two of whom were at low risk and one at high risk of being affected from analysis of linked DNA markers) did not have an abnormal band; the fourth patient had an abnormal band of $+0.3 \mathrm{~kb}$ as detected by Southern blotting which was not amplified in the PCR reaction. Clinical examination of these four patients had shown that one patient with a normal eye examination had an EMG examination which was reported to be diagnostic of myotonic dystrophy, while the remaining three had polychromatic lens crystals on slit lamp examination of the eye and normal EMG, one of whom was the subject with the abnormal band.

For the three subjects from group II, one was found to have an abnormal band of $+0.5 \mathrm{~kb}$ and results have not yet been obtained for the other two. These three patients had not been examined by EMG, but were said to have clinical evidence of apparent weakness, and two were said to have demonstrable myotonia and to have also developed bilateral cataract in their early 50s. The subject with the abnormal band did not have myotonia or bilateral cataract.

For the single subject in group III an abnormal band of $+0.7 \mathrm{~kb}$ was detected. For the two subjects in group IV, one clearly has no abnormal band and the second has not been typed; however, the spouses of both these subjects have shown abnormal bands by PCR.

The four subjects with normal alleles on PCR and no abnormal band on Southern blot

Table 2 Sensitivity of EMG in relation to molecular analysis.

\begin{tabular}{llr}
\hline EMG analysis & Genotype & No \\
\hline Normal & N/N & 1 \\
Normal & N/DM & 11 \\
Abnormal & N/N & 1 \\
Abnormal & N/DM & 7 \\
No of patients with EMG analysis & & 20 \\
Sensitivity of EMG $=7 / 18=39 \%$ & &
\end{tabular}

$\mathrm{N} / \mathrm{N}$ two alleles in normal size range.

$\mathrm{N} / \mathrm{DM}$ one allele in normal size range plus one in DM size range.

Table 3 Sensitivity for ophthalmic signs in this series.

\begin{tabular}{lcc}
\hline Cataract or polychromatic lens crystals & Genotype & No \\
\hline Absent & N/N & 0 \\
Absent & N/DM & 1 \\
Present & N/N & 3 \\
Present & N/DM & 39 \\
Total no of patients analysed & 43 \\
Sensitivity of ophthalmic signs $=39 / 40=97.5 \%$ &
\end{tabular}

Sensitivity of ophthalmic signs $=39 / 40=97.5 \%$

$\mathrm{N} / \mathrm{N}$ two alleles in normal size range.

$\mathrm{N} / \mathrm{DM}$ one allele in normal size range plus one in DM size range. analysis are thus likely to be homozygous for a normal allele at the myotonic dystrophy locus.

\section{Discussion}

While the clinical and electrophysiological evidence on patients in groups I and II appears highly suggestive of them carrying the myotonic dystrophy mutation, normal molecular analysis in seven of these underlines the need for cautious interpretation of non-specific clinical signs in a patient at risk of carrying the gene.

Clear clinical false positive diagnoses are indicated by those patients in groups I and II who have two normal PCR alleles. There are four such patients out of the 56 ( 38 males and 18 females, $7 \%$ ) tested. Patient 1 is a 28 year old clinically normal man who did not have a positive EMG, but had bilateral polychromatic lens crystals on the basis of which he had been misdiagnosed as being positive for myotonic dystrophy. Patient 2 is a clinically normal 20 year old woman with polychromatic lens crystals and a normal EMG examination who had been inappropriately diagnosed on the basis of her eye findings. Patient 3 is a 70 year old man with bilateral cataract who did not have EMG but reported leg weakness with stumbling and falling and whose clinical examination showed percussion myotonia. Patient 4 is a 65 year old woman with a normal slit lamp examination but whose EMG showed myopathic features. However, none of the typical features of myotonic dystrophy was apparent. Clinical examination was reported to show extensive facial, neck, and lower limb weakness, on the basis of which she had been afforded disease status.

Two patients in group III had normal clinical parameters but were thought to be at high risk by linked DNA markers. Molecular analysis showed that they both had two normal alleles confirming that they were unaffected. In each instance the assumption of phase in the family had been erroneous.

The assignation of status in all eight families in group IV was complete, with the origin of the abnormal allele identified in all cases. Seven abnormal alleles were identified from this cohort of patients among whom rigorous clinical investigation had failed to indicate the gene carriers. Two subjects were heterozygous for normal alleles, four having a partner with an abnormal band and the partner of the fifth being dead. Two subjects had one normal allele only; however, in both instances their partner's analysis showed an abnormal allele, indicating true homozygosity of a normal allele. Based on the allele frequencies detected in the normal population, ${ }^{11}$ subjects have a 1 in 3 chance of being homozygous for the (CTG) polymorphism if they are normal, whereas all DM carriers should be heterozygous (one normal allele and one DM specific allele) unless their abnormal band is too large to be detected under the PCR conditions described.

The size of the abnormal alleles in group IV ranged from 53 to 60 triplet repeats and is the smallest so far detected in the disease range in 
our series. Allele size for patients with or without muscle disease (groups I and II) appears broadly similar with a range of 53 to 160 triplet repeats and a median of 70 . Thus, there is no evidence in this study to suggest that the presence of mild muscle disease presenting in the fifth decade or later indicates a larger allele size at the disease locus. However, these data derive from lymphocyte DNA and different levels of amplification may occur in other tissues. The data from groups III and IV emphasise the value of this molecular analysis as a diagnostic test in asymptomatic, clinically and electrophysiologically normal gene carriers.

In this series, eight patients with EMG findings characteristic of myotonic dystrophy were examined, seven of whom have clearly defined abnormal alleles. Similarly, 12 patients with negative EMG findings were examined, of whom 11 have an abnormal allele and one had two normal alleles. Thus the sensitivity for EMG is $39 \%$ (table 2). Specificity was not calculated because patients had been selected as being minimally expressing gene carriers and therefore the number of normal non-gene carriers was too small to be meaningful.

The same approach to ophthalmic signs of eye disease in patients said to have minimal expression of the gene based on their ophthalmic findings shows a sensitivity of $97.5 \%$ (table 3). It must be emphasised that these data relate to the minimally expressing cohort only and the sensitivity of clinical, ophthalmic, and electrophysiological examination in patients at $50 \%$ risk of carrying the myotonic dystrophy gene has already been put at 90 to $92 \% .^{67}$ Nevertheless, these data reinforce the unreliability of clinical parameters alone in assigning gene carrier status, especially in minimally expressing cases of myotonic dystrophy.

We thank Mrs Lyn Beck for performing ophthalmic assessment of many of the patients in this study, and Drs Iain MacQueen and Gerry Heath for EMG studies. Our work is supported by the Muscular Dystrophy Group of Great Britain, the Medical Research Council, the Muscular Dystrophy Association of America, and the Wellcome Trust.

1 Curschmann $H$. Uber familiare atrophische Myotonie. Dtsch Z Nervenheilkd 1912;45:161-202.

Dtsch $Z$ Nervenheilkd 1912;45:161-202.
2 Fleischer B. Uber myotonische Dystrophie mit Karakt. Arch Klin Ophthalmol 1918;96:91-133.

Arch Klin Ophthalmol 1918;96:91-133.
3 Harper PS. Myotonic dystrophy. 2nd ed. London: Saunders, 1989.

4 Dyken PR, Harper PS. Congenital dystrophia myotonica. Neurology 1973;23:465-73.

5 Norman AM, Floyd JL, Meredith AL, Harper PS. Presymptomatic detection and prenatal diagnosis for myotonic dystrophy by means of linked DNA markers. $f$ Med Genet 1989;26:750-4.

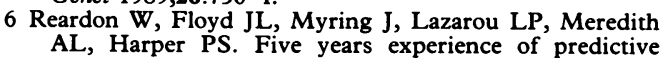
testing for myotonic dystrophy using linked DNA markers. Am ₹ Med Genet 1992;43:1006-11.

7 Brunner HG, Smeets HJM, Nillesen W, et al. Myotonic dystrophy. Predictive value of normal results on clinical examination. Brain 1991;114:2303-11.

8 Harley HG, Brook JD, Rundle SA, et al. Expansion of an unstable DNA region and phenotype variation in myounstable DNA region and phenotype varia
tonic dystrophy. Nature 1992;355:545-6.

9 Buxton J, Sheibourne P, Davies J, et al. Detection of an unstable fragment of DNA specific to individuals with unstable fragment of DNA specific to individur
myotonic dystrophy. Nature 1992;355:547-8.

10 Aslanidis C, Jansen G, Amemiya C, et al. Cloning of the essential myotonic dystrophy region and mapping of the putative defect. Nature 1992;355:548-51.

11 Brook JD, McCurrach ME, Harley HG, et al. Molecular basis of myotonic dystrophy: expansion of a trinucleotide (CTG) repeat at the $3^{\prime}$ end of a transcript encoding a protein kinase family member. Cell 1992;68:799-808.

12 Harley HG, Rundle SA, Reardon W, et al. Unstable DNA sequence in myotonic dystrophy. Lancet 1992;339:1125-

13 Samb.

3 Sambrook J, Fritsch EF, Maniatis T. Molecular cloning-a Laboratory Press, 1989. 